\title{
Complex Formation Between Alkaline-Earth Cations and Anthraquinone Crown Ethers in Methanol and Acetonitrile
}

\author{
J.M. Caridade Costa, ${ }^{a, *}$ P.M.S. Rodrigues ${ }^{b}$ \\ ${ }^{a}$ Departamento de Química, Universidade de Coimbra, 3004-535 Coimbra, Portugal. \\ ${ }^{b}$ Instituto Politécnico da Guarda, P-6300 Guarda, Portugal.
}

Received 5 April 2004; accepted 20 May 2004

\begin{abstract}
Formation of complexes of alkaline-earth cations with crown ethers containing an anthraquinone unit (AQ18C6 and AQ21C7) was investigated in methanol and acetonitrile solutions. Stability constants of the resulting complexes were estimated by means of potentiometric methods. The results obtained show the formation of complexes of the $\mathrm{LM}^{2+}$ type for all alkaline-earth ions; for the larger cations, $\left(\mathrm{Sr}^{2+}\right.$ and $\left.\mathrm{Ba}^{2+}\right)$ and in excess of ligand, $\mathrm{L}_{2} \mathrm{M}^{2+}$ complexes were also detected. The most stable complexes were obtained with $\mathrm{Ba}^{2+}$ cation and for both ligands. The binding strength for the cations towards AQ18C6 and AQ21C7 ligands and in both solvents, increased in the order, $\mathrm{Mg}^{2+}<\mathrm{Ca}^{2+}<\mathrm{Sr}^{2+}<\mathrm{Ba}^{2+}$. The results of this study suggest that on the $1: 1$ complexes, the size of the cation and its fit into the macrocyclic internal cavity of the ligand was a dominant factor on the coordination binding. The number of donor atoms in the ring of the macrocycle does not affect the binding strength and the AQ18C6 complexes have larger stability than the corresponding AQ21C7 complexes. The presence of an anthraquinone unit on the structure of the crown ether induced a decrease of the cation binding strength. The external carbonyl group of the ligand molecules was not involved on the coordination to the cation. Considering the two solvents, the complexes were more stable on acetonitrile, the solvent of weaker solvating capacity.
\end{abstract}

Keywords: alkaline-earth cations, crown ether derivatives, complexation, potentiometric methods.

\footnotetext{
* Corresponding author. E-mail address: caridade@ci.uc.pt
} 


\section{Introduction}

Crown ethers and their derivatives are synthetic molecules whose features have constituted an important field of investigation over the last few decades. The binding and selective affinity with metal ions in solution is one of their most relevant properties and has led to many applications in chemistry and biochemistry [1-5].

The complexation of metal ions and their selective recognition by molecular receptors containing a structure of the crown ether type includes the direct contact between the two chemical species. This process involves in sequential order: desolvation (total or partial) of the cation and ligand, interaction between cation and ligand, and often structural reorganisation in the ligand $[3,6,7]$. Some or all of these steps can be of great relevance to understand and determine important properties such as binding stability and selective transport of ions across natural or synthetic membranes, and control of analytical separation processes [8-12].

The selective binding process of a metal ion requires by the host cavity of a macrocyclic ligand, appropriate dimensions, shape and structural characteristics, in such a way that in the complexed form, the available donor atoms can be organised to provide the most favourable coordination geometry $[6,13]$.

The macrocyclic compounds can yet be modified with the purpose to improve their capacity to act as selective agents towards metal ions. The change in the dimension of the macrocyclic ring, the modification in the arrangement, nature and number of complexing atoms in the ring, or the incorporation in the appropriate position of side arms with donor groups, are some of the alterations that can change the stability of the complexes and possibly improve the selectivity of the ligands for one metal ion over the others $[6,11,14,15]$.

In this paper we report the complexing ability of two synthetic ligands constituted by a cyclic polyether incorporating an anthraquinone unit towards alkaline-earth cations, in two non-aqueous solvents, methanol and acetonitrile, by using potentiometric methods. 
The molecules studied in this work are of special interest due to the presence of a quinone group and a crown ether unit. After electrochemical reduction, these molecules induce a stronger interaction with the cation as a result of the coupling of a redox process to the cation coordination process, as was previously shown $[16,17]$.

With this study we also wish to obtain data on free energy that together with the changes in enthalpy and entropy, will allow us to discuss the thermodynamic aspects that can control and explain the complexation of the alkaline-earth cations with this type of ligands.

Selective electrodes that can be used on potentiometry for a direct measurement of the concentration of free alkaline-earth cations in organic solvents were not available. Thus, we have used competition reactions with the silver ion as the competitive ion (and a silver ion-selective electrode), to calculate the complexation constants.

The several factors that can influence the complexation stability will be analysed in the perspective of the capacity of this type of ligands to coordinate to the alkaline-earth cations.

\section{Experimental}

The alkaline-earth salts used were analytical grade (Alfa) and dried under vacuum pump for 3 days at room temperature prior to use. AQ18C6 and AQ21C7 compounds were synthesised and purified as was previously described [16]. Tetrabutylammonium perchlorate (TBAP, Fluka), was twice recrystalized from ethyl acetate, followed by drying in an oven at $100{ }^{\circ} \mathrm{C}$ for $24 \mathrm{~h}$ under vacuum and stored in a desiccator. Acetonitrile (reagent grade for chromatography, Merck) and methanol (HPLC quality grade, Lab-Scan) were used without further purification.

The stability constants of the complexes of the alkaline-earth cations were obtained by a potentiometric method based on a competition for the ligand by the alkaline-earth cation and silver cation [18]. The formation constant of the silver complex combined with the effect of the silver ion on the ligand-alkaline earth 
ion equilibrium, allows to calculate the required stability constants. Details of the potentiometric titrations have already been described [19].

Values of the constants for the complexation of the silver ion with each ligand are first determined independently [19]. Titrations to calculate the stability constants of the alkaline-earth ions are then performed in a solution containing the ligand and the alkaline-earth cation to which is added the silver cation. The characteristic potential of the free silver concentration is monitored at each titration point after the complexation equilibrium has been established using two different cells. In acetonitrile solutions, we used a cell constituted by a silver/silver chloride electrode of double junction (Orion, model 90-02) as reference, and a silver metal electrode (Methrom, model 6.0331.010) as indicator. In methanol solutions, we employed the same reference electrode and a selective electrode of membrane of silver/silver sulphide (Orion, model 96-14) as indicator.

The potentiometric titrations were performed in the range of concentrations, $\mathrm{C}_{\mathrm{L}}=$ $1.8 \times 10^{-4}-2.1 \times 10^{-3} \mathrm{~mol} \mathrm{dm}^{-3} ; \mathrm{C}_{\mathrm{Ag}}=4.0 \times 10^{-6}-7.2 \times 10^{-3} \mathrm{~mol} \mathrm{dm}^{-3} ; \mathrm{C}_{\mathrm{M}}=1.2 \times$ $10^{-3}-8.7 \times 10^{-3} \mathrm{~mol} \mathrm{dm}^{-3}$.

To ensure the consistency of the results, we have calculated the stability constants of the $18 \mathrm{C} 6$ molecule with the sodium and potassium cations in methanol and acetonitrile solutions, according to the method previously described. A good agreement was obtained between our determinations and the published data [20,21].

\section{Results and discussion}

This study shows the effect of complexation in the reactions between AQ18C6 and AQ21C7 ligands (L) (Fig. 1) and the alkaline-earth cations $\left(\mathrm{M}^{2+}\right)$ in methanol and acetonitrile solutions.

The treatment and refinement of the potentiometric data in order to determine the stability constants and type of species formed were made using the Superquad program, [22] introducing the necessary modifications to adopt this computer program to our data systems and strategy. In the present study and under the 
experimental concentrations used, it is assumed that the ionic association effects were not significative for the calculated stability constants [23].

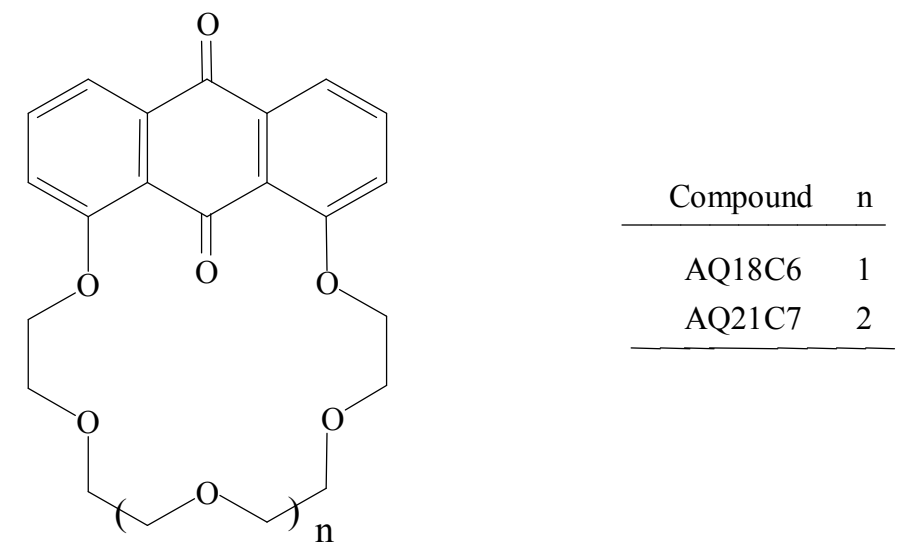

Figure 1. Chemical structure of the molecules, AQ18C6 $(n=1)$ and AQ21C7 $(n=2)$.

Under the experimental conditions used, the data treatment revealed 1:1 $\left(\mathrm{LM}^{2+}\right)$ species on all systems; for the larger cations, $\mathrm{L}_{2} \mathrm{M}^{2+}$ complexes were also formed, as shown in Tables 1 and 2 .

The stability of the complexes of both ligands follows the sequence, $\mathrm{Ba}^{2+}>\mathrm{Sr}^{2+}$ $>\mathrm{Ca}^{2+}>\mathrm{Mg}^{2+}$. This indicates that the thermodynamic stability of the metal ionligand complexes does not depend only on the ion-dipole interactions, but strongly reflects the influence of the species size and consequent fitting of the cation into the macrocyclic internal cavity of the ligand. This is the main reason that leads to the larger stability constants and to the higher selectivity for the larger metal cations over the smaller ones. Fig. 2 and 3 show the correlation between cation size and complex stability.

Any of the alkaline-earth cations can be placed inside the macrocyclic internal cavity of both ligands, but $\mathrm{Ba}^{2+}$ (diameter=2.70 $\AA$ ) [24] is the cation of larger size and smaller charge density, which induces the highest binding stability in the alkaline-earth cation series. This cation has the most suitable size, among the alkaline-earth cations, to fit into the cavity of the AQ18C6 molecule (size 2.77 A) [20] and is also the one that better approaches the dimension of the macrocyclic cavity of AQ21C7 (size@3.40 A) [20], thus inducing the stronger cation-oxygen binding. Owing to its good fitting into the macrocyclic cavity of 
the AQ18C6 molecule, the $\mathrm{Ba}^{2+}$ cation should occupy a central position relatively to the plane of the macroring atoms. The symmetric and planar geometry of the complex induces a more efficient participation of all the electrodonor atoms on the coordination and a more favourable conformational energy of the macrocyclic ligand [14,25].

Table 1. Stability constants $(\log \beta)$ for complexes of alkaline-earth metal ions with AQ18C6 and AQ21C7 ligands in methanol solution. $\left(\mathrm{t}=25^{\circ} \mathrm{C}, \mathrm{I}=0.1 \mathrm{~mol} \mathrm{dm}{ }^{-3}\right.$ TBAP).

\begin{tabular}{|c|c|c|c|c|}
\hline \multirow{2}{*}{$\begin{array}{l}\text { Metal } \\
\text { ion }\end{array}$} & \multicolumn{2}{|c|}{ AQ18C6 } & \multicolumn{2}{|c|}{$\mathrm{AQ} 21 \mathrm{C} 7$} \\
\hline & $\log \beta_{11}$ & $\log \beta_{21}$ & $\log \beta_{11}$ & $\log \beta_{21}$ \\
\hline $\mathrm{Mg}^{2+}$ & $2.86 \pm 0.08$ & - & $2.51 \pm 0.06$ & - \\
\hline $\mathrm{Ca}^{2+}$ & $3.08 \pm 0.05$ & - & $2.71 \pm 0.05$ & - \\
\hline $\mathrm{Sr}^{2+}$ & $3.44 \pm 0.06$ & $8.30 \pm 0.13$ & $3.08 \pm 0.08$ & $6.24 \pm 0.17$ \\
\hline $\mathrm{Ba}^{2+}$ & $4.08 \pm 0.05$ & $8.52 \pm 0.10$ & $3.67 \pm 0.08$ & $7.27 \pm 0.15$ \\
\hline
\end{tabular}

(a) Values were determined with the selective electrode of membrane of $\mathrm{Ag} / \mathrm{Ag}_{2} \mathrm{~S}$ using a competitive potentiometric titration.

(b) $\beta_{11}$ and $\beta_{21}$ refer to the complexation equilibrium, $\mathrm{L}+\mathrm{M}^{2+} \leftrightarrow \mathrm{LM}^{2+}$ and $2 \mathrm{~L}+\mathrm{M}^{2+} \leftrightarrow \mathrm{L}_{2} \mathrm{M}^{2+}$, respectively, where $\mathrm{M}^{2+}$ is the cation and $\mathrm{L}$ is the ligand molecule.

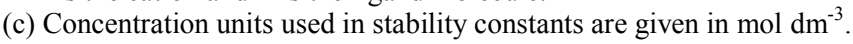

Table 2. Stability constants $(\log \beta)$ for complexes of alkaline-earth metal ions with AQ18C6 and AQ21C7 ligands in acetonitrile solution. $\left(\mathrm{t}=25^{\circ} \mathrm{C}, \mathrm{I}=0.1 \mathrm{~mol} \mathrm{dm}^{-3}\right.$ TBAP).

\begin{tabular}{|c|c|c|c|c|}
\hline \multirow{2}{*}{$\begin{array}{c}\text { Metal } \\
\text { ion }\end{array}$} & \multicolumn{2}{|c|}{ AQ18C6 } & \multicolumn{2}{|c|}{ AQ21C7 } \\
\hline & $\log \beta_{11}$ & $\log \beta_{21}$ & $\log \beta_{11}$ & $\log \beta_{21}$ \\
\hline $\mathrm{Mg}^{2+}$ & $3.81 \pm 0.03$ & - & $2.95 \pm 0.04$ & - \\
\hline $\mathrm{Ca}^{2+}$ & $3.92 \pm 0.04$ & - & $3.34 \pm 0.06$ & - \\
\hline $\mathrm{Sr}^{2+}$ & $5.37 \pm 0.04$ & $10.86 \pm 0.09$ & $3.93 \pm 0.08$ & $7.61 \pm 0.13$ \\
\hline $\mathrm{Ba}^{2+}$ & $6.93 \pm 0.07$ & $14.10 \pm 0.13$ & $4.30 \pm 0.09$ & $8.28 \pm 0.18$ \\
\hline
\end{tabular}

(a) Values were determined with the silver metal electrode using a competitive potentiometric titration.

(b) $\beta_{11}$ and $\beta_{21}$ refer to the complexation equilibrium, $\mathrm{L}+\mathrm{M}^{2+} \leftrightarrow \mathrm{LM}^{2+}$ and $2 \mathrm{~L}+\mathrm{M}^{2+} \leftrightarrow \mathrm{L}_{2} \mathrm{M}^{2+}$, respectively, where $\mathrm{M}^{2+}$ is the cation and $\mathrm{L}$ is the ligand molecule.

(c) Concentration units used in stability constants are given in $\mathrm{mol} \mathrm{dm}^{-3}$.

On the other hand, $\mathrm{Mg}^{2+}$ cation forms the least stable complex among the alkaline-earth cations due to its most polarising character. In what concerns the fit of both the macrocyclic internal cavity and $\mathrm{Mg}^{2+}$, this cation has a size (diameter=1.14 $\AA$ ) [24] which is substantially smaller than that of the cavity of the AQ18C6 ligand. Some of its oxygen atoms have more difficulty to associate 
with appreciable force to that cation in a planar conformation. Possibly not all of the potential donor atoms in the ligand may effectively interact with $\mathrm{Mg}^{2+}$, as this ion introduces some intramolecular repulsion in the macrocyclic structure thus weakening the strength of interaction with the cation [26,27].

The $\mathrm{Ca}^{2+}$ and $\mathrm{Sr}^{2+}$ cations induce intermediate binding strengths between $\mathrm{Ba}^{2+}$ and $\mathrm{Mg}^{2+}$ with both AQ18C6 and AQ21C7 ligands as a result of their intermediate ionic sizes, with the $\mathrm{Sr}^{2+}$ complexes more stable.

Another factor that influences the formation and stability of the complexes is the solvation change of the participating species in the complexation. Investigation to separate the free energy of complexation in enthalpic and entropic components is in progress and will be shortly reported.

The presence and participation of one oxygen atom of a carbonyl group in the macrocyclic internal cavity in appropriate geometrical orientation with the cation binding centre can also influence the coordination. This probably leads to the smaller cations being positioned on the plane of the macroring, in a nonsymmetrical position relatively to the potential donor atoms of the macrocyclic cavity [26]. As a consequence, in the coordination of the smaller cations we can consider that conformational changes occur in the complexed ligand structure. These conformational changes are more unfavourable to the complex stability since they bring the ligand out of its own equilibrium conformations [25].

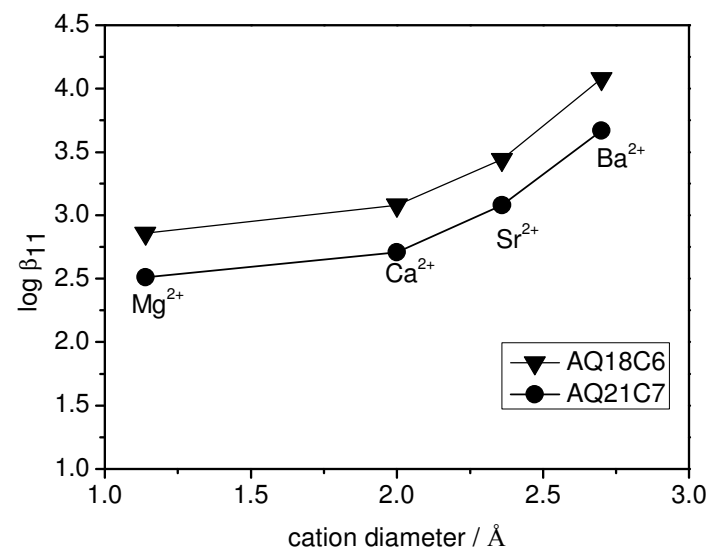

Figure 2. Correlation between the cation size and stability of the 1:1 complexes of the AQ18C6 and AQ21C7 ligands in methanol solution. 
The results obtained seem to indicate that the carbonyl oxygen atom positioned out of the macrocyclic ring does not participate on the coordination. Effectively, the distance and mainly the orientation of its lone pairs of electrons places in a remote position for interaction with any of the cations.

A decrease in the complexation strength of all cations was obtained when another $-\mathrm{OCH}_{2} \mathrm{CH}_{2}$ - unit was introduced into the macrocyclic cavity (AQ21C7), as is observed in Fig. 2 and 3. In spite of the increase in the number of potential donor atoms that participate on the binding, the relative complexation affinity is smaller with AQ21C7 than with AQ18C6 for each cation. A likely reason can be the increase in the distances between each oxygen atom and the cation and also a less favourable orientation of the lone pair electrons of the donor atoms of AQ21C7. On the other hand, it is possible that the ligand with more appropriate cavity size (AQ18C6) is able to adopt a more stable and efficient conformational donor atoms of AQ21C7. On the other hand, it is possible that the ligand with more appropriate cavity size (AQ18C6) is able to adopt a more stable and efficient conformational geometry and this may also be reflected in part on the differences of affinity verified between the two ligands for each cation [6].

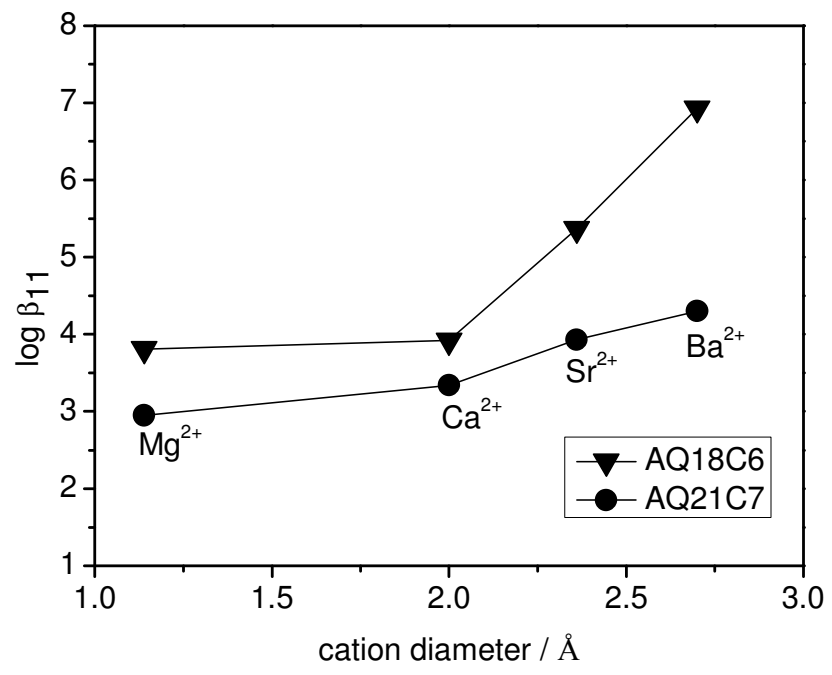

Figure 3. Correlation between the cation size and stability of the 1:1 complexes of the AQ18C6 and AQ21C7 ligands in acetonitrile solution. 
It is interesting to verify that in methanol there is an almost constant decrease in the complex stability $\left(\Delta \log \beta_{11} \cong 0.37\right)$ relatively to each cation when we go from AQ18C6 to AQ21C7 (Fig. 2). In acetonitrile solution, the difference in the stability is not constant, but increasingly larger when we go from the complexes of the smaller cations to the larger cations (Fig. 3).

In all systems studied an 1:1 complex $\left(\mathrm{LM}^{2+}\right)$ was determined. However for the cations with larger sizes $\left(\mathrm{Ba}^{2+}\right.$ and $\left.\mathrm{Sr}^{2+}\right)$ a $\mathrm{L}_{2} \mathrm{M}^{2+}$ complex was also evidenced and for the two ligands. Fig. 4 shows a species distribution diagram for the system $\mathrm{Ba}^{2+} / \mathrm{AQ18C6}$. As observed, the formation of $\mathrm{L}_{2} \mathrm{Ba}^{2+}$ complex is significative when there is an excess of ligand, having a structure in which the cation makes the bridge between two ligand molecules. For larger concentrations of $\mathrm{Ba}^{2+}$ relatively to the AQ18C6, $\mathrm{LBa}^{2+}$ becomes increasingly dominant.

As shown in Tables 1 and 2, the stability of the complexes is influenced by the solvating ability of the solvent, and the largest stability constants for each cation are associated to the acetonitrile, the solvent of weakest solvating ability. The differences of stability observed on the formed complexes in the two solvents, acetonitrile and methanol, increase in general with the increasing of the cation size.

We verified that the stability of the complexes of the AQ18C6 and AQ21C7 molecules with alkaline-earth cations is lower than that corresponding to unsubstituted crown ethers [20]. This decrease of the stability constants can be attributed to steric and inductive effects induced by the anthraquinone unit. Effectively, the presence of benzo groups in the ligands reduces the basicity of the oxygen atoms that are beside it and may induce a more limited conformational flexibility of the molecule cavity and a decrease in its size $[21,28]$, leading to the thermodynamic weakening of the interaction cationligand.

Considering the ions with comparable size, $\mathrm{Na}^{+}$and $\mathrm{Ca}^{2+}$, and $\mathrm{K}^{+}$and $\mathrm{Ba}^{2+}$, the complexes of the alkaline-earth are more stable than the alkali cations [19]. From these results we can conclude that, in addition to the effects of size and fit, the charge density of the complexed cation also contributes to the complexation 
stability. The electrostatic nature of the binding between the oxygen atoms and cation and the stronger solvation of the alkaline-earth cations relatively to the alkali cations, lead to a larger stability of the first over the last [29].

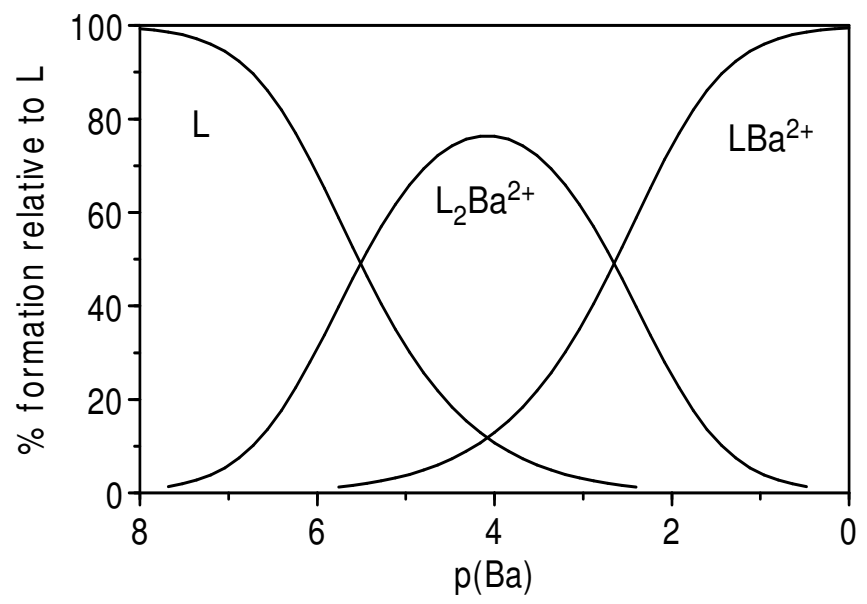

Figure 4. Species distribution observed in the complexation of $\mathrm{Ba}^{2+}$ cation with AQ18C6 ligand in methanol solution $\left(\mathrm{C}_{\mathrm{L}}=10^{-3} \mathrm{~mol} \mathrm{dm}^{-3}\right)$.

\section{Conclusions}

The results of this study have revealed that the stability of the alkaline-earth cation complexes is largely influenced by the size of the macrocyclic internal cavity and cation. The better the fitting of the cation into the cavity, the stronger the interactions between the cation and the donor atoms of the ligand, as is observed for $\mathrm{Ba}^{2+}$ cation, which forms the most stable complexes among the alkaline-earth cations with both ligands. The smaller cation, $\mathrm{Mg}^{2+}$, formed the less stable complexes.

The results have indicated that the binding strength on the complexation will also have an important contribution from the combination of electrostatic and structural factors.

All the alkaline-earth cations formed 1:1 complexes; $\mathrm{Sr}^{2+}$ and $\mathrm{Ba}^{2+}$ (the larger cations) also formed 2:1 complexes when there is a ligand excess in solution.

The ligand with the smaller number of potential binder atoms formed the complexes of higher stability when compared with the ligand with larger number 
of donor atoms, especially because the donor atoms are at a smaller distance from the complexed cation.

The external carbonyl oxygen of the macrocyclic ring does not participate in the binding to the cation because of its position and because of the orientation of its binding electrons.

The inclusion of a neutral anthraquinone unit in the crown ether structure decreased the stability of the complexes relatively to the corresponding crown ether unsubstituted.

\section{References}

1. D.J. Cram, Angew. Chem. Int. Ed. Engl. 25 (1986) 1039.

2. $\quad$ R.A. Bulman, Struct. Bonding 67 (1987) 91.

3. R.D. Hancock and A.E. Martell, Chem. Rev. 89 (1989) 1875.

4. L.F. Lindoy, The Chemistry of Macrocyclic Ligand Complexes, Cambridge University Press, 1989.

5. Y. Umezawa (Ed.), Handbook of Ion-Selective Electrodes: Selectivity Coefficients, CRC Press, Boca Raton, Florida, 1990.

6. R.M. Izatt, K. Pawlak, J.S. Bradshaw and R.L. Bruening, Chem. Rev. 95 (1995) 2529.

7. V.P. Solov'ev, N.N. Strakhova, O.A. Raevsky, V. Rüdiger and H.J. Schneider, J. Org. Chem. 61 (1996) 5221.

8. J.M. Lehn, Angew. Chem. Int. Ed. Engl. 27 (1988) 89.

9. Y. Inoue and G.W. Gokel, (Eds.), Cation Binding by Macrocycles: Complexation of Cationic Species by Crown Ethers, Marcel Dekker, New York, 1990.

10. H.C. Visser, D.N. Reinhoudt and F. de Jong, Chem. Soc. Rev. 23 (1994) 75.

11. J.S. Bradshaw and R.M. Izatt, Acc. Chem. Res. 30 (1997) 338.

12. P. Bühlmann, E. Pretsh and E. Bakker, Chem. Rev. 98 (1998) 1593.

13. V.J. Gatto, K.A. Arnold, A.M. Viscariello, S.R. Miller, C.R. Morgan and G.W. Gokel, J. Org. Chem. 51 (1986) 5373.

14. B. Dietrich, J. Chem. Ed. 62 (1985) 954. 
15. G.W. Gokel, Chem. Soc. Rev. 21 (1992) 39.

16. J.M. Caridade Costa, J. Jeyashri and D. Bethell, J. Electrochem. Soc. 351 (1993) 259.

17. J.M. Caridade Costa and D. Bethell, J. Coord. Chem. 46 (1999) 551.

18. D.A. Gustowski, V.J. Gatto, J. Mallen, L. Echegoyen and G.W. Gokel, J. Org. Chem. 52 (1987) 5172.

19. J.M. Caridade Costa and P.M.S. Rodrigues, Port. Electrochim. Acta 20 (2002) 167.

20. J.D. Lamb, R.M. Izatt, C.S. Swain and J.J Christensen, J. Am. Chem. Soc. 102 (1980) 475.

21. H.J. Bushmann, J. Sol. Chem. 17 (1988) 277.

22. P. Gans, A. Sabatini and A. Vacca, J. Chem. Soc., Dalton Trans. 1195 (1985).

23. H.J. Buschmann, E. Cleve and E. Schollmeyer, J. Sol. Chem. 23 (1994) 569.

24. R.D. Shannon, Acta Crystallogr. A32 (1976) 751.

25. B.G. Cox, P. Firman and H. Schneider, Inorg. Chim. Acta 69 (1983) 161.

26. P.D.J. Grootenhuis and P.A. Kollman, J. Am. Chem. Soc. 111 (1989) 2152.

27. E. Karkhaneei, J. Zolgharnein, A. Afkhami and M. Shamsipur, J. Coord. Chem. 46 (1998) 1.

28. Y. Takeda, Y. Ohyagi and S. Akabori, Bull. Chem. Soc. Jpn. 57 (1984) 3381.

29. H.J. Buschmann, J. Sol. Chem. 15 (1986) 453. 